\title{
Le leitmotiv du défi dans les autobiographies des handicapés: Application sur Les jours de Taha Hussein et Le cri de la mouette d'Emmanuelle Laborit.
}

Saddam Mohammed Ahmed Khalil

\section{Introduction.}

Sans doute, le défi apparaît comme un leitmotiv dans les genres littéraires ayant comme un thème le handicap et les handicapés. Dans cette étude, nous abordons la configuration du défi dans deux autobiographies écrites par deux auteurs handicapés. Le défi se représente comme un état de résilience en face de tous les obstacles et les lourdeurs engendrées par handicap. Les handicapés qui ne cessent pas de souffrir, surtout dans des sociétés qui les marginalisent et les humilient explicitement et implicitement, recourent au défi en volonté de fer en vue de récompenser tout manque orienté à leurs personnalité ou à leurs qualités. Taha Hussein présent un modèle inoubliable du défi puisque sa vie était pleine de souffrance, de problèmes, et d'obscurité. Il combat la pauvreté et l'ignorance. Il consiste à aboutir au succès en débit d'accusations d'échec et de défaite qu'il recevait de son père, de son frère, et de ses cheikhs à l'université d'El-Azhar. D'autre part, nous prenons comme un autre exemple l'auteure sourde Emmanuelle Laborit qui, malgré son handicap, sera comédienne puisque c'est son rêve le plus cher. Adolescente révoltée Emmanuelle va lutter, souffrir et finalement remporter

(*) Maître-assistant au Département de Français, Faculté Al-Alsun, Université de Sohag.

Cette recherche fait une partie d'une thèse doctorat, intitulée: "L'image du handicapé dans Les jours de Taha Hussein et Le cri de la Mouette d'Emmanuelle Laborit: Étude comparée", Sous la direction du. Dr. Hanna Abdel-Ghany Hamouda, Professeur émérite de la littérature française, Faculté des Lettres. Université de Minia \& Dr. Taha Roshdy Taha, Professeur adjoint de la linguistique française et Chef de département de Français, Université de Kafr El-sheik. 
son victoire : devenir actrice, mais aussi plaider la cause de millions de malentendants. Pour que le monde des sourds ne soit plus le monde du silence.

Mots clés: handicapé, défi, révolte, souffrance résilience.

Nous divisons notre étude en quelques points essentiels comme suit:

-Définition du handicapé.

-Définition de l'autobiographie comme genre littéraire envahi par les handicapés

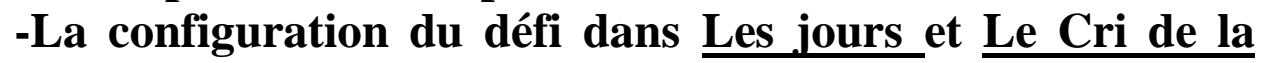
mouette

\section{-Conclusion}

\section{- Définition du handicapé.}

Selon Le petit Ropert, «Handicapé est la personne qui présente un handicap physique ou mental» ${ }^{l}$. La loi n²005-102 pour l'égalité des droits et des chances, la participation et la citoyenneté des personnes handicapées a, dans son article 114, défini la notion de handicap: "toute limitation d'activité ou restriction de participation à la vie en société subie dans son environnement par une personne en raison d'une altération substantielle, durable ou définitive d'une ou plusieurs fonctions physiques, sensorielles, mentales, cognitives ou psychiques, d'un polyhandicap ou d'un trouble de santé invalidant. " ${ }^{2}$. Selon Larousse le handicapé est «Personne atteinte d'une infirmité ou défavorisée sur un point quelconque» ${ }^{3}$.

-Définition de l'autobiographie comme genre littéraire envahi par les handicapés

Nous ne pouvons pas entamer une telle étude sans présenter une définition concise de l'autobiographie en essayant de dévoiler tout ce qui se cache sous ce terme expressif et complexe. Le terme se compose de trois mots comme suit:

Auto; une notion provenant du mot grec "autos", c'est-à dire, " soi-même, lui-même". Bio; une notion provenant aussi du mot grec "bios", c'est-à-dire "la vie". Graphie; une notion grecque qui veut dire " écrire". L'autobiographie est d'un 
caractère herméneutique, c'est-à-dire l'art de l'interprétation. L'écrivain écrit le soi, il réduit la distance d'éloignement envers son soi en utilisant l'écriture comme moyen qui réalise un parcours.

Selon Georges Gusdorf Auto: "c'est l'identité, le moi conscient de lui-même » ${ }^{4}$, C'est un sujet complexe qui s'est lentement élaboré au cours d'une existence particulière et autonome. Bio, c'est le parcours vital, la continuité, le cheminement de cette identité unique et singulière : la variation existentielle autour du thème fondamental que constitue l'auto, le moi, c'est-à-dire entre auto et bio se trace en révélant un rapport difficile de l'être et de son existence, de l'identité et de la vie, rapport que tout le monde connaît entre individualité et déroulement pratique d'une existence, entre le moi et son inscription dans la réalité. Quant à la graphie, c'est l'activité scripturaire et la possibilité d'entamer une nouvelle vie ${ }^{5}$

La longueur et la subtilité du terme peut révéler plusieurs indications et significations comme la longueur de la période de l'écriture car le narrateur écrit une vie complète en commençant par sa naissance ${ }^{6}$ jusqu'au moment d'enregistrer, ou dès le moment qui a véritablement touché sa mémoire en passant par les détails, en ralentissant parfois et en accélérant d'autrefois et ainsi de suite d'une manière qui manifeste un grand effort.

"L'autobiographe vise à figurer l'existence dans sa totalité ou du moins jusqu'au moment où il écrit. Mais il s'agit moins de l'appréhender dans sa durée totale que dans sa signification globale. L'autobiographe ne raconte pas seulement les événements de la vie, il s'efforce de les ordonner, d'en trouver la logique secrète, de les rapporter à des causes. Il veut montrer comment il est devenu ce qu'il est et se l'expliquer à lui-même. La forme du récit continu lui sert à constituer sa propre histoire comme un processus linéaire» ${ }^{7}$

Ce terme peut indiquer aussi à la complexité des vérités et des événements racontés et leurs imbrications les uns dans les autres, et finalement peut indiquer à la spécificité et à la 
possession des récits et des polémiques racontés aux personnes narrateurs: "c'est un rapport complexe qui pose la représentation comme projet réalisable et la réalisation de la représentation comme projet impossible ${ }^{8}$. Et c'est justement qu'on remarque dans la définition de Philippe Lejeune qui définit l'autobiographie comme un "Récit rétrospectif en prose qu'une personne réelle fait de sa propre existence, lorsqu'elle met l'accent sur sa vie individuelle, en particulier sur l'histoire de sa personnalité » ${ }^{9}$. Cependant, la définition de l'autobiographie semble et reste confuse car il s'agit d'une relativité véritable soit pour les intentions de l'écriture, soit du niveau de véridicité: "question d'authenticité et non d'exactitude ${ }^{10}$, soit du choix d'un style ou d'un ton précis. En outre, les nouveautés ne se termineront pas car c'est l'art de s'exprimer notamment à l'ombre des bouleversements qui touchent les natures humaines en engendrant des motifs et des intentions incontournables.

\section{- La configuration du défi dans Les jours et Le Cri de la mouette.}

Tant de cas handicapés ne tentent pas de réaliser un succès sous prétexte du handicap. Selon Pierre Villey «Dans la réalité, aussi bien qu'un stimulant, la perte d'un sens peut être un stupéfiant pour l'organisme; au lieu d'affiner les autres sens, elle peut avoir pour effet de les émousser»"

Discuter le thème du défi dans Les jours et Le Cri de la mouette n'est pas ni choix ni une redondance ni une exposition facultative qu'on peut denier ou ignorer, mais il apparaît obligatoire pour contourner un tel thème basé surtout sur une question d'une approche sociale. Le motif du défi peut s'accorder ou se différencier dans les deux ouvrages, mais les résultats sont similaires enfin puisque le défi a mené au succès dans les deux cas. Les deux autobiographies exige une relecture d'une vision qui s'intéresse à dégager les traits de défi car c'est à travers le défi qu'apparaît une image peu parfaite du handicapé et des obstacles engendrés à l'issu de ce handicap, de la 
souffrance résistée ou défiée, de succès réalisé malgré ces souffrances, des procédés suivis pour atteindre au succès et des valeurs véritables soient positives soient négatives exigeant le défi.

Taha Hussein et Emmanuelle Laborit présentent un considérable exemplaire de défi parce que leur défi est contre des conditions trop difficiles et trop exceptionnelles. Le défi de Taha Hussein est un leitmotiv, dès son enfance, puisqu'il apparaît d'une personnalité rebellé et résistante. Le défi d'Emmanuelle Laborit apparaît finalement dans sa démarche, c'est-à-dire après avoir tenté de suicider, de dévier et de s'isoler à part. D'autre part, pour les deux, ce défi devient le point de départ jusqu'à l'apogée de leur succès immédiat. Ils ont réussi à fonctionner leurs possibilités limites en s'emballant la négativité qui touchent un grand nombre de leurs similaires. Ils éprouvent une réalité absente pour beaucoup de gens même pour ceux qui entendent; c'est le manque absolu, c'est-à-dire personne n'est pas complet mais il y a ceux qui ont de la confiance en leurs potentialités et leurs compétences.

Le défi de Taha Hussein est toujours lié à son handicap, voire au succès réalisé malgré ce handicap. C'est Taha Hussein qui résiste en recevant ses droits par insistance à l'instruction et au fonctionnement, au voyage, au mariage: «"Taha Hussein a vécu une vie de luttes constantes - politiques, sociales et personnelles» ${ }^{12}$. Son défi sert aussi à corriger les conceptions envers les aveugles d'une manière qui modifie nos conduites et nos croyances car selon Pierre Villey "Le clairvoyant croit que l'aveugle reste écrasé du fardeau qui l'accable, que les sources mêmes de la personnalité en lui sont empoisonnées. C'est là une impression chez le clairvoyant plus qu'un jugement, je le sais, mais aussi nos impressions pèsent elles plus dans notre conduite que nos jugements» ${ }^{13}$. C'est pourquoi, un tel défi de la part des handicapés apparaît doublé, premièrement envers euxmêmes, puis envers leurs entourages. Nous insistons que le défi de Taha Hussein est à l'issue d'une bonne foi et d'une forte 
croyance qui le font résistant et souriant et ne se laisse pas aux chagrins en réalisant l'action de défier: "Louange à Allah que ce garçon n'a pas soumis à la tristesse et que ses circonstances ne lui ont pas poussé au désespoir, en revanche, il a continué son chemin autant qu'il a pu, en essayant le bien pour lui-même et pour les gens» ${ }^{14}$.

En affrontant sa cécité, et en défiant ses vœux intérieurs, il a relevé "une volonté de fer" selon sa propre expression. Il a évité et délaissé volontairement toutes les situations et les choses qui peuvent lui engendrer des problèmes, par exemple, il a délaissé les sortes de la nourriture qui révèlent son infirmité et n'acceptait pas l'aide de quelqu'un. C'est une sorte de satisfaction qui montre un défi contre les plus simples exigences et plaisirs.

"C'était depuis cet instant qu'il se découvrir une volonté de fer. Il s'interdit à lui-même certaines nourritures dont il ne goûta qu'après avoir dépassé vingt-cinq ans. Il condamna les potages et le riz, et tous les plats qui se mangent à la cuillère, car il se savait inhabile avec cet instrument ${ }^{15}$.

De même, cette volonté définitive est le moteur principal à sa célébrité comme il est enregistré dans le livre intitulé: The Sufferers: Stories and Polémics, «Par sa propre volonté et son désir de connaissance, il a grandi pour devenir le leader de la renaissance culturelle arabe» ${ }^{16}$. Son défi était plus fort que son infirmité qui lui apparaît toujours comme un monstre, il traitait toutes ses affaires patiemment d'une manière qui a fait de cette infirmité comme une source d'énergie et de résistance: "Nous ne pouvons pas ignorer les avantages de son handicap, c'est à travers son handicap qu'il a se mis à prendre des situations indépendantes» ${ }^{17}$. Une volonté très forte qui le poussait hardiment au défi, sa vie n'était jamais pleine de roses. À cause de sa cécité, il envisageait des pressions et des conflits plus que les voyants mais il n'abandonnait jamais ni ses rêves ni ses désirs. Il est le conquérant de l'impossible comme le nommait Kamal Hussein qui lui a consacré un livre intitulé Dr. Taha 
Hussein; le conquérant de l'obscurité: «Nombreux sont les fois dont Taha Hussein a échoué ! Mais personne n'est plus vite que lui à dépasser l'échec en la transformant à un succès! Il a pu transformer le désespoir à une sorte de défi qu'il affrontait et $n^{\prime} y$ échappait pas» ${ }^{18}$.

Il a connu la résistance en défiant son père, les hôtes de son père, et Sayedna lorsqu'il a échoué trois fois à réciter le Coran en prétendant qu'il l'a su par cœur. Il a déployé tous ses efforts pour réaliser le succès désiré et pour reprendre la confiance de son entourage. Il a véritablement su le Coran en dépassant leur examen. De plus, il a su l'Alfiyya ${ }^{19}$ d'Ibn Malek $^{20}$ et beaucoup d'autres sciences en dépit de Sayedna et son père qui ne connaissent que le Coran et qui le menacent plus qu'une fois d'interrompre son enseignement.

Taha Hussein n'a pas connu ni l'impossible ni l'ennui ni la défaite. Il avait une insistance irrésistible et n'acceptait rien sans être persuadé par les épreuves et les justifications. Il insistait son opinion au point que son adversaire se retirait dans un état indescriptible de la défaite. Prenons par exemple sa rage contre le cheikh qui prétendait la science alors qu'il n'avait pas le moindre et préfère le retrait.

" [...] Colère $d u$ jeune homme et riposte ardente $d u$ professeur. "Un long bavardage n'a jamais consacré une vérité ni supprimé une erreur ", lança le jeune homme. Le cheikh se tut et ce fut un silence général, que le maître rompit : "Vous pouvez disposer, ça suffit pour aujourd'hui ${ }^{21}$.

Son défi apparaît de plus envers sa cécité et envers le système abusif de l'Université qui a directement refusé, au début, de l'envoyer pour la France sous prétexte de son infirmité. Il n'a pas hésité de répéter son requête plus qu'une fois. De sa part, l'Université a mis des obstacles paralysants pour le faire se détourner à son but. Le conseil a proposé, d'abord, l'ajournement de la bourse, puis, il a mis comme condition la connaissance parfaite de la langue française, et enfin, et pour que Taha Hussein renonce tout à fait à son désir, 
il a mis comme condition la réalisation d'une thèse de doctorat. En face de son insistance, en utilisant toutes les possibilités et en dépassant tous les examens, l'Université n'a pas pu négliger son droit légal de bénéficier d'une de deux bourses gouvernementales présentées dans ce temps-là: "Rien ne lui fit plus de plaisir que de relever à son tour le défi. Il se mit donc à préparer cette épreuve avec soin et à rédiger sa thèse...» ${ }^{22}$.

Taha Hussein ne délaissait jamais l'un de ses souhaits, il vainquait dans tous les polémiques et les discussions qui lui s'imposaient. Il tente d'échapper de la prison de la cécité en réalisant un succès et une célébrité sociale. Selon Fedwa MaltiDouglas: "La prison est cependant non seulement physique mais aussi sociale. C'est en brisant les barreaux sociaux que Taha échappe à sa prison d'aveuglement.» ${ }^{23}$. Il était comme un conflit intérieur par lequel il a dépassé la honte qu'il subissait de son entourage puisqu'il est né au sein d'un village où les gens cachent leurs enfants handicapés en les considérant comme une source de l'humiliation, Fedwa Malti-Douglas voit aussi que "Les concepts d'aveuglement dans une société affecteront les rôles attribués aux personnes visiblement handicapées et influenceront à leur tour la façon dont un individu perçoit sa propre cécité» ${ }^{24}$. Alors, ayant ses principes fixes et clairs, Taha Hussein a vécu toute sa vie en état du défi durable contre l'ignorance, l'injustice, la discrimination, les entraves de la vie...etc.

Quant à Emmanuelle Laborit, son premier défi se représente à travers sa fierté d'être sourde contrairement à ceux qui se cachent ou souffrent la honte. Elle déclare, par moquerie, que vouloir que tous les enfants, à la naissance, ne soient plus « sourds », c'est vouloir un monde parfait. Comme si on les voulait tous blonds, aux yeux bleus, etc. Elle ne cesse de s'interroger pourquoi ne pas accepter les imperfections des autres ? Elle résiste la croyance à l'invalidité des sourds. Il se moque en tout défi: "Par rapport à vous, entendants, Emmanuelle est imparfaite. Il faut naître avec des oreilles qui 
entendent, une bouche qui parle. Pareille. Les autres entendent, pas moi. Mais j'ai mes yeux, ils observent bien mieux que les vôtres, forcément» 25.

Une telle parole sévère d'une sourde dans une société basée sur la parole est un défi. La volonté de créer un état d'union contre la mutation imposée est un défi contre tous ceux qui croient à leur manque. Si Emmanuelle n'a pas résisté l'état de marginalisation dont vivent les sourds, ils pourraient vivre encore sans aucun rapport avec leur entourage, ils n'obtiendraient pas aucune législation à pratiquer la plupart de leurs droits car ils perdent l'outil principal de se communiquer et s'exprimer. Selon René Kaës "C'est la parole qui fonde ce qui nous lie les uns aux autres dans l'humanité» ${ }^{26}$. Son défi est visé à elle-même avant tout, elle devrait croire sa question en vue de posséder la force et la qualité de défendre. Elle ne cesse de réfléchir en vue de comprendre les codes des choses. Elle insiste d'essayer en déployant tous ses efforts à réaliser son succès.

"Je lis des tas de journaux, je bouquine jusqu'à n'y voir plus clair. J'ai la tête farcie de tellement de choses que je dois avoir l'air complètement abrutie, par moments. C'est dans ma nature de me dépasser, d'aller au bout des choses que j'entreprends. Quand je décide d'atteindre un but, je n'abandonne pas. Rien, ou presque rien ne m'arrête. Mouette têtue.» ${ }^{27}$.

Emmanuelle Laborit donne une épreuve sans précédent à tous ceux qui apparaissent peu démoralisés ou paresseux soient handicapés ou naturels. Une volonté qui nous fait remémorer la volonté de fer de Taha Hussein malgré le manque de ses capacités ou ses possibilités, elle encourage en outre à travailler sans ennui. Il veut savourer la réussite qui peut lui donner des motivations positives et des sentiments compensatoires. Elle montre que seule la volonté réelle et la patience qui poussent à dépasser l'échec. Parfois la peur de perte devient un motif primordial à réussir, c'est justement son cas. Sa surdité ne l'empêche pas de rêver la célébrité et de déployer ses efforts 
pour l'atteindre. Il sait qu'une telle société estime les hommes célèbres de science, de la littérature, de l'art et des autres catégories bien cultivées et bien instruites. Elle s'accorde tout à fait avec le cas de Taha Hussein qui a évalué l'enseignement jusqu'à devenir l'un de ses pionniers. Elle déclare que son défi passe par des étapes difficiles et accablantes. Elle passe son temps dans les dictionnaires et les bouquins pour trouver précisément le sens d'une phrase qu'elle a retenue sur les lèvres d'un professeur. Elle potasse les cours. Elle bosse parfois jusqu'à deux ou trois heures du matin, comme une dingue. Le bilinguisme lui aide énormément: "Et je veux toujours faire de belle construction de phrase, en français, avoir un beau style. Parce que je le voudrais académique, impeccable» ${ }^{28}$. C'est pourquoi elle ne cesse de frapper les portes où elle peut bénéficier; utiliser la langue des signes malgré son interdiction au début, compter sur ses parents et sa sœur la moins âgée qu'elle, employer d'aidants technologiques comme le Minitel. Elle sait qu'est ce qu'elle veut en précisant son but et en demandant de ses parents de fournir les outils convenables. C'est elle qui reconnaît que son niveau ne s'adapte pas avec l'étude secondaire et pour résoudre un tel problème, elle recourt aux cours privés par correspondance en vue de pouvoir récupérer les cinquante pour cent manquants de la géo, la philo, l'histoire, le français, l'anglais, la bio et le reste. Pour ne pas se décourager complètement, pour s'accrocher à ce "Passe ton bac d'abord ${ }^{29}$. Elle aime le défi et peut travailler sous des pressions pour atteindre son rêve. Il faut noter qu'elle cherche tôt l'indépendance, c'est pourquoi elle est si sérieuse. Il ne se voit pas inhabile de pratiquer des travaux comme le font les entendants. Âgée dix-sept ans, elle se met à gagner son bain à travers plus qu'un métier dont leur nature s'adapte avec ses compétences: "J'ai besoin de me refaire une santé. Je trouve des petits boulots, du baby-sitting comme toutes les jeunes filles. Garder des petits enfants, ça me fait du bien. Ça me ramène à mon enfance» ${ }^{30}$. 
"Dépasser ton bac" est la condition mise par la famille pour lui permettre d'atteindre le théâtre. Sa carrière au théâtre a commencé par une petite pièce qui s'appelait Voyage au bout du métro. Le théâtre était un soleil dans sa vie d'enfant: «Le soleil qui part du cœur $»^{31}$. Le théâtre a rempli son esprit résistant. Sa pièce de théâtre principale a évoqué son enthousiasme surtout le thème de résistance ou de défi qu'elle représente: "Les Enfants du silence racontent le défi de deux mondes. Celui d'un entendant, Jacques, et celui de Sarah, sourde. C'est une histoire de révolte, d'amour, d'humour» ${ }^{32}$. Elle a su comment vivre en équipe, avec les comédiens; Affrontements, disputes ententes, amour, entendants et sourds mêlés, c'est un échange extraordinaire, précieux. Ainsi configure-t-elle le défi. Laissons-nous Emmanuelle Laborit parle de son succès et sa gloire qui apparaît comme un résultat de son défi: "On m'a demandé de participer à des émissions de télévision, j'ai visité toutes les chaînes. On me propose des rôles pour le cinéma. Tout va si vite, je suis dans un tourbillon» ${ }^{33}$

Elle semble si fière et si heureuse que tout ce monde des médias s'intéresse, à travers elle, au monde du silence. Elle a réalisé une transition miraculeuse en se transformant de l'état de marginalisation à la célébrité répandue partout. La société ne connaît pas les sourds. Elle ne cesse de donner l'impression de révéler au monde entier qu'ils existent. Ils sont gentils, habiles, talentueux, adorables, passionnés, attentifs et admiratifs: "Tous les sourds se reconnaissent en Emmanuelle Laborit; elle est leur ambassadrice, leur porte-parole incontestable.» ${ }^{34}$. Nous conclusions ce discours désignant le défi par son épreuve lorsqu'elle a décidé d'enregistrer son autobiographie dans un livre pour être un témoin et un guide à la fois. Hélas, elle a rencontré des essais de démoralisation psychologique et de déception qu'elle a affrontés rigoureusement ${ }^{35}$.

\section{Conclusion.}

A travers cette étude, les handicapés sont apparus d'un véritable rôle au niveau social, intellectuel et littéraire. Nous 
avons découvert que le défi accomplit les sentiments du manque sentis par les handicapés, que les handicapés affrontent parfois les difficultés d'une patience et d'une volonté de dépasser les crises, les entourages sociaux sont ceux qui poussent les handicapés à adopter le défi contre les normes et les croyances injustes. Les sociétés, à la différence de leurs cultures et leurs lois, peuvent mettre des obstacles qui menacent le succès des handicapés.

Pour les handicapés, l'acte de s'écrire à travers une autobiographie se représente à la tête des aspects du défi puisqu'ils s'annoncent volontairement en toute force pour laisser des témoignages à leurs similaires. Dans leurs autobiographies, ils dénoncent la société, la pauvreté, l'ignorance, la discrimination et la marginalisation, ils donnent des épreuves et des règles du défi non seulement pour les handicapés, mais aussi pour les naturels.

\section{Références}

${ }^{1}$ ROBERT, (Paul), "Le petit Robert", Canada, Montréal, Le Robert, 1996, p.346

${ }^{2}$ Enquête Handicap-Incapacités-Dépendance, Résultat n ${ }^{\circ} 6$ INSEE, Septembre 2002.

3 [En Ligne]

http://www.larousse.fr/dictionnaires/francais/handicap\%C3\%A9/38990? $\mathrm{q}=$ handicap\%C3\%A9\#38917

${ }^{4}$ GUSDORF (Georges), Lignes de vie 2 Auto-bio-graphie, Odile Jacob, Paris, 1991, p.10

${ }^{5}$ CF., MIRAUX (Jean Philippe), L'autobiographie: écriture de soi et sincérité, Paris, Nathan Université, 1996, (Coll.«128».), sommaire de l'ouvrage, envoyé par Myvin, p.4, disponible sur https://www.google.com.eg/url? sa $=t \& r c t=j \& q=\& e s r c=s \&$ source $=w e b \&$ $\mathrm{cd}=1 \& \mathrm{cad}=\mathrm{rja} \& u a c t=8 \& \mathrm{ved}=0 \mathrm{ahUKEwia} 9 \mathrm{c} 661 \mathrm{OjYAhVHYlAKHa} 4 \mathrm{~d}$ DocQFggmMAA\&url=http\%3A\%2F\%2Ffabyanaa.chez.com\%2F128_a utobio_Myvin.doc\&usg=AOvVaw1Mc9Xe8Xa_1NK-2r47khl3

${ }^{6}$ Personne ne se rappelle les détails de sa propre naissance, mais les individus de sa famille peuvent lui raconter après.

${ }^{7}$ JENNY (Laurent), la figuration de soi, Cours et méthodes, Genève, 2003 in www.unige.ch, 
${ }^{8}$ MIRAUX (Jean Philippe), Op., Cit., p.15

${ }^{9}$ LEJEUNE (Philippe), Le pacte autobiographique, Paris, Seuil, 1997, p.

${ }^{10}$ MIRAUX (Jean Philippe), Op., Cit., p.15

${ }^{11}$ VILLEY (Pierre), La pédagogie des aveugles, Paris, FélixAlcan, 1993, p.8

${ }^{12}$ HUSSEIN (Taha), The Sufferers: Stories and Polemics, (Modern

Arabic writing), The American University in Cairo Press 1993, introduction, traduit de l'anglais par nous.

${ }^{13}$ VILLEY (Pierre), Le monde des aveugles: Essai de psychologie, Paris, Flammarion, 1914. p.4

${ }^{14}$ HUSSEIN (Taha), Le livre des jours, Gallimard, La France, 1947 p.9

${ }^{15}$ Loc. Cit

${ }^{16}$ ID, The Suffreres: Stories and Polemics, introduction.

${ }^{17}$ KAREM (Sameh), Que reste de Taha Hussein? (Mazaa tabaquaa mn Taha Hussein), Le Caire, Dar el-kotob, 1975, p.19, traduit de l'arabe par nous.

${ }^{18}$ HUSSEIN (Kamal), Dr/ Taha Hussein, Vainqueur de l'obscurité, Le Caire, Alahram, 1973, p.139, traduit de l'arabe par nous.

${ }^{19}$ Célèbre grammaire de l'arabe classique

${ }^{20}$ Auteur de l'Alfiyya

${ }^{21}$ HUSSEIN (Taha), Le livre des jours, p.237

${ }^{22}$ Enfin, il se présenta à cet examen et obtint le titre de docteur [...]

Celui-ci ne trouva pas le sommeil. Il avait relevé et gagné le défi qui lui avait été lancé; il était à présent docteur de l'Université. Son voyage en France était dès lors une dette que celle-ci devrait bien penser, tôt ou tard, à honorer »._HUSSEIN (Taha), La traversée intérieure, préface d'Etiemble, Gallimard, 1992.p.77-78.

${ }^{23}$ MALTI-DOUGLAS (Fedwa), Blindness and Autobiography: AlAyyam of Taha Husayn, University press, Princeton, New Jersey, 1988, p.15, traduit de l'anglais par nous

${ }^{24}$ Ibid., p. 16

${ }^{25}$ J'ai mes mains qui parlent. Un cerveau qui accommode des informations, à ma manière, selon mes besoins. Je ne vais pas vous traiter d'imparfaits, vous, les entendants. D'ailleurs, je ne me le permettrais pas. Au contraire, je veux l'union entre les deux communautés, avec le respect. Je vous donne le mien, j'attends le vôtre LABORIT(Emmanuelle), Le Cri de la mouette, éditions Pocket Jeunesse, département D'Univers Poche, pour la présente édition, Paris, 2003, p.319 
${ }^{26}$ KAËS (René), «Enfance», Vo.60, 2008, Éditeur : Presses

Universitaires de France

${ }^{27}$ LABORIT(Emmanuelle), Op., Cit., p.306

${ }^{28}$ Ibid., p.304

${ }^{29}$ Ibid., , p.307

${ }^{30}$ Ibid., p.266.

${ }^{31}$ Ibid., p. 163

${ }^{32}$ Ibid., p.339

${ }^{33}$ Et pendant ce temps, nous sillonnons la France avec "Les Enfants du silence". Chaque soir, je frémis en saluant le public, en voyant les mains se lever pour applaudir. «J'entends » le succès. Il vibre dans tout mon corps. La petite lumière rouge du téléphone ne cesse de clignoter. Il y a tant de projets dans la vie de la mouette. Tant de choses à faire, à dire, à jouer. A aimer. LABORIT (Emmanuelle), Op., Cit., p.300

${ }^{34}$ BOROY(Annie), Mes enfants sourds: Langue française et intégration Le pari du Langage Parlé Complété, Editions L'Harmattan 5-7, rue de l'Ecole Polytecnique, 75005 Paris, S.D, p.9

35 «Lorsque j'ai voulu faire ce livre, certaines personnes m'ont dit: «Tu n'y arriveras pas. » $\mathrm{Oh}$ ! Si. Quand je décide de faire quelque chose, je vais jusqu'au bout. Je voulais y arriver. J'avais décidé d'y arriver» LABORIT(Emmanuelle), Op., Cit, p.15 